Results: Twenty eight patients were enrolled in the study. They were 19 women (sex-ration $\mathrm{M} / \mathrm{F}=0.6$ ), with a mean age of 54 years [extremes: 13 - 73 years]. Most patients had type $1 \mathrm{AlH}(89.2 \%)$. Seventeen patients were diagnosed at stage of cirrhosis $(60.7 \%)$. Associated auto-immune manifestations were observed in $42.8 \%$ of cases. Overlap syndrome with primary biliary cirrhosis was noted in $21.4 \%$ of cases. Fifty five percent of patients were on steroid treatment with or without azathioprine. BMD was low in 9 patients $(32 \%)$ as fellow: osteopenia in 6 cases and osteoporosis in 3 cases. There was a correlation between bone loss and use of steroid treatment but it wasn't statistically significant $(p=0.07)$.

Conclusions: In our series, the prevalence of bone loss in AlH is high (45\%). This data suggests that bone status should be assessed routinely in patients with $\mathrm{AlH}$, especially in those on steroid treatment.

Disclosure of Interest: None declared

DOI: 10.1136/annrheumdis-2017-eular.6442

\section{AB0834 RISK FACTORS FOR DECREASED BONE MINERAL DENSITY IN INFLAMMATORY BOWEL DISEASE IN A TUNISIAN COHORTE}

H. Romdhane ${ }^{1}$, M. Cheikh ${ }^{1}$, K. Abdelghani ${ }^{2}$, R. Ennaifer ${ }^{1}$, N. Bel Hadj ${ }^{1}$, A. Laatar ${ }^{2} .{ }^{1}$ Gastroenterology and hepatology; ${ }^{2}$ Rhumatology, Mongi Slim Hospital, TUNIS, Tunisia

Background: Patients with inflammatory bowel disease [IBD] are at risk for metabolic bone disease. Many studies have identified various risk factors but most of them have involved western patients.

Objectives: The aim of this study was to investigate the prevalence and the risk factors for metabolic bone disease in Tunisian IBD patients.

Methods: Retrospective study including patients with IBD admitted in our department between January 2011 and December 2015. Demographic and clinical characteristics of patients were analysed. Bone mineral density of the femoral neck, total femur and lumbar spine was quantified by dual-energy X-ray absorptiometry.

Results: Among 82 patients followed for IBD $(70.7 \%$ with Crohn's disease; $29.3 \%$ with Ulcerative colitis), a bone densitometry was performed in $56 \%$ of cases $(n=46) .16$ patients have osteopenia and 7 had osteoporosis, as assessed by T-score. Univariate analysis showed that Crohn'n disease in particular ileal disease, high steroid dose and the presence of extra-intestinal manifestations were significantly associated with a low bone mineral density (for all $p<0.05$ ). In the other hand, IBD duration since diagnosis, sexe, tabagism were not associated with bone loss.

In multivariate regression analysis, risk factors for decreased bone mineral density were IBD duration since diagnosis, high steroid dose, ileal crohn's disease and extra-intestinal manifestations.

Conclusions: In our Tunisian cohort of IBD patients, Crohn's disease, high steroid dose and extra-intestinal manifestations were associated with increased risk for metabolic bone disease. High risk patients should be identified and appropriate therapies should be started early to improve long term quality of life.

Disclosure of Interest: None declared

DOI: 10.1136/annrheumdis-2017-eular.5301

\section{AB0835 DENOSUMAB AS A FIRST CHOICE DRUG FOR GLUCOCORTICOID INDUCED OSTEOPOROSIS TREATMENT INSTEAD OF BISPHOSPHONATE}

I. Yoshii ${ }^{1}$, T. Chijiwa ${ }^{2} .{ }^{1}$ Orthopaedic Surgery, Yoshii Hospital, Shimanto City; ${ }^{2}$ Rheumatology, Kochi Memorial Hopsital, Kochi, Japan

Background: Glucocorticoid induced osteoporosis (GIO) is serious problem for raising risk of bone fragile fracture. In general, first choice drug for $\mathrm{GIO}$ is bisphosphonate (BPH), however, denosumab (dMAB), a monoclonal antibody of receptor activator of nuclear factor kappa-B ligand, is closed up as a alternative selection for $\mathrm{GIO}$ recently.

Objectives: The aim of this study is to evaluate effectiveness of dMAB in bone mineral density (BMD) for GIO treatment compared to $\mathrm{BPH}$.

Methods: In the patients in whom glucocorticoid steroid (GCs) have been administered for more than three months, who met indication criteria for GIO what was determined by the Japanese Society for Bone and Mineral Research in 2014, that is matrix calculated in adding points of past fracture history, age, dosage of GCs, and BMD value (1), were enrolled. Before March 2013, data was lacking, so patients who have been administrated GCs after April 2013 were picked up. Patients BMD at GCs administration, at 6 months after initial treatment, if drug was changed, also at 6 months after second treatment, for minimum lumbar spine (LS), femoral neck (FN), and greater trochanter (GT) were measured with dual-energy X-ray absorptiometry (DEXA). Patients were classified by drug for initial treatment and second drug if administered. Patients age, initial, average, and total dose of GCs, term length of administration, and BMD and its gain for each chance were compared with Mann-Whitney U-test and Student's paired T-test.

Results: 149 patients in whom 48 with no drug administrated (N), 24 for BPH naïve and continued (BB), 22 for $B P H$ naïve and changed to $\mathrm{dMAB}(\mathrm{BD}), 21$ for $\mathrm{DMAB}$ naïve and continued (DD), 34 for dMAB naïve and changed to $\mathrm{BPH}$ (DB) were counted. In these, sex distribution was 26 for men and 123 for women. Underlying disease for administration of GCs were rheumatoid arthritis for 114 , polymyalgia rheumatica for 12 , idiopathic thrombocytopenic purpura for
9, systemic lupus erythematosus for 6 , and others for 8 . For groups, age at baseline, initial, average, and total dose, and term length of administration of GCs demonstrated no significant difference between any pairs of the groups. BMD at baseline for Group N demonstrated significant greater per-cent of young adult mean (\%YAM) than Group DD $(p<0.01)$ in all parts, yet greater than the other groups but not statistically significant. In Group N, BMD had significantly decreased from the baseline to 6 months later in all parts $(p<0.01)$. In the other groups, BMD had shown gain at 6 months after drug administration in all part, however, in Group DB showed mean \%YAM loss for GT after first and second drug administration compared to Group BD had shown \%YAM loss after first but gain after second drug for FN (Table 1).

\begin{tabular}{|c|c|c|c|c|c|}
\hline First Drug (FD) & none & \multicolumn{2}{|c|}{ bisphosphonate } & \multicolumn{2}{|c|}{ denosumab } \\
\hline Second Drus (SD) & none & none & denosumab & none & bisphosphonate \\
\hline Cases & 48 & 24 & 22 & 21 & 34 \\
\hline age at baseline & $69.7 \pm 10.8$ & $73.7 \pm 16.5$ & $81.8 \pm 122$ & $72.5 \pm 11.5$ & $71.5 \pm 10.8$ \\
\hline initial dose of GCs & $6.78 \pm 8.98$ & $6.88 \pm 8.25$ & $5.58 \pm 6.95$ & $5.21 \pm 3.47$ & $6.95 \pm 2.25$ \\
\hline average dose of GCs & $3.77 \pm 2.13$ & $5.49 \pm 3.76$ & $5.06 \pm 1.61$ & $3.49 \pm 1.49$ & $5.12 \pm 2.06$ \\
\hline term length of administration & $53.6 \pm 45.3$ & $84.7 \pm 44.9$ & $38.2 \pm 46.4$ & $51.9 \pm 58.7$ & $65.2 \pm 46.0$ \\
\hline total dose of $\mathrm{GCs}_{\mathrm{s}}$ & $3836.6 \pm 4444.3$ & $7170.2 \pm 10923.8$ & $2447.1 \pm 6365.9$ & $5017.1 \pm 9057.3$ & $7898.2 \pm 772.9$ \\
\hline BMD of lumbar spine at baseline & $89.1 \pm 16.9$ & $78.1 \pm 17.7$ & $80.2 \pm 124$ & $70.9 \pm 9.3$ & $75.9 \pm 12.4$ \\
\hline BMD of LS at six months after administration of FD & $86.5 \pm 19.1$ & $79.3 \pm 10.5$ & $81.8 \pm 15.2$ & $72.9 \pm 13.5$ & $75.9 \pm 15.5$ \\
\hline BMD of LS at six months after administration of SD & $2<$ & $2<$ & $81.2 \pm 12.8$ & $2<$ & $77.3 \pm 9.2$ \\
\hline BMD of FN at baseline & $90.8 \pm 12.8$ & $77.3 \pm 9.3$ & $70.6 \pm 12.9$ & $66.8 \pm 7.6$ & $72.1 \pm 8.8$ \\
\hline $\mathrm{BMD}$ of $\mathrm{FN}$ at six months after administration of $\mathrm{FD}$ & $87.8 \pm 15.4$ & $80.0 \pm 14.6$ & $69.1 \pm 122$ & $65.9 \pm 12.4$ & $74.3 \pm 11.4$ \\
\hline BMD of $F N$ at six months after administration of SD & $2<$ & 2 & $71.2 \pm 11.3$ & $>$ & $74.4 \pm 15.3$ \\
\hline BMD of GT at baseline & $85.8 \pm 9.8$ & $80.8 \pm 10.1$ & $71.2 \pm 12.7$ & $67.1 \pm 12.6$ & $75.2 \pm 9.4$ \\
\hline BMD of GT at six months after administration of FD & $83.5 \pm 14.5$ & $80.1 \pm 12.9$ & $71.2 \pm 12.7$ & $68.9 \pm 12.6$ & $75 \pm 9.3$ \\
\hline$B M D$ of $G T$ at six months after administration of SD & $\sum<$ & $\sum<$ & $74.2 \pm 13.3$ & $\sum<$ & $74.2 \pm 10.3$ \\
\hline
\end{tabular}

Conclusions: From these results, dMAB is effective role in raising BMD for GIO as a initial drug, and a second drug even after inadequate response to $\mathrm{BPH}$. dMAB could be possible to be chosen as a first choice drug for GIO treatment. References:

[1] Suzuki, Y., Nawata, H., Soen, S. et al. J Bone Miner Metab (2014) 32: 337. doi:10.1007/s00774-014-0586-6.

Disclosure of Interest: None declared

DOI: 10.1136/annrheumdis-2017-eular.3106

\section{AB0836 PERFORMANCE OF QUANTITATIVE ULTRASOUND AND SIX OSTEOPOROSIS RISK INDEXES IN MENOPAUSAL WOMEN: VALIDATION AND COMPARATIVE EVALUATION STUDY}

I. Ghozlani ${ }^{1}$, A. El Maataoui ${ }^{2}$, M. Ghazi ${ }^{3}$, A. Kherrab ${ }^{3}$, R. Niamane ${ }^{3}$. ${ }^{1}$ Rheumatology Department, Military Hospital; ${ }^{2}$ Chemistry-Biochemistry Department, Faculty of Medicine and Pharmacy, Ibn Zohr University, Agadir; ${ }^{3}$ Rheumatology Department, Military Hospital Avicenne, Marrakech, Morocco

Background: A number of questionnaire-based systems and the use of portable quantitative ultrasound scanners (QUS) have been devised in an attempt to produce a cost-effective method of screening for osteoporosis.

Objectives: to assess the sensitivity and specificity of different techniques and their ability to act as screening tools in relation to dual energy $\mathrm{X}$-ray absorptiometry (DXA).

Methods: 295 white postmenopausal women aged over 60 were enrolled. Each subject completed a standardized questionnaire which permits the measure of six osteoporosis indexes and had bone mineral density (BMD) measured using QUS and DXA. Sensitivity and specificity of the different techniques in relation to DXA were plotted as receiver-operating characteristic (ROC) curves at DXA T-score total hip $\leq-2.5$ (osteoporosis).

Results: BUA sensitivity and specificity values were respectively $76.8 \%$ and $51.2 \%$ at the total hip. The optimal cut-off T-score for QUS was -2 at the total hip. The osteoporosis self-assessment tool (OST) provided consistently the highest AUC $(0.80)$ among the clinical tools and had the best sensitivity and specificity balance $(90.2 \%>44.5 \%)$. OST negative likelihood ratio was 0.22 .

Conclusions: OST (based only on the weight and the age) performed slightly better than QUS and other risk questionnaires in predicting low BMD at the total hip.

Disclosure of Interest: None declared

DOI: 10.1136/annrheumdis-2017-eular.1449

\section{AB0837 BONE METABOLISM AND OSTEOPOROSIS RISK FACTORS ANALYSIS IN SPINAL CORD INJURY PATIENTS AT TWELVE MONTHS FOLLOW UP}

I. Martínez Cordellat ${ }^{1}$, F. Torralba Collados ${ }^{2}$, C. Grao Castellote ${ }^{2}$, E. Grau García $^{1}$, V. Fornes Ferrer ${ }^{3}$, E. Labrador Sánchez ${ }^{1}$, K. Arévalo Ruales ${ }^{1}$, J. Fragio Gil ${ }^{1}$, R. González Mazarío ${ }^{1}$, C. Alcañiz Escandell ${ }^{1}$, I. Chalmeta Verdejo ${ }^{1}$, C. Feced Olmos ${ }^{1}$, L. González Puig ${ }^{1}$, J. Ivorra Cortés ${ }^{1}$

R. Negueroles Albuixec ${ }^{1}$, J.E. Oller Rodríguez ${ }^{1}$, F. Ortiz Sanjuán ${ }^{1}$, E. Bernabeu $V_{\text {Vicens }}{ }^{1}$, C. Nájera Herranz ${ }^{1}$, I. Cánovas Olmos ${ }^{1}$, J.A. Román Ivorra ${ }^{1}$

${ }^{1}$ Rheumatology Department; ${ }^{2}$ Spinal Cord Injury Unit; ${ }^{3}$ Biostatistics Unit IIS Ia $\mathrm{Fe}$, Hup la Fe, Valencia, Spain

Background: The spinal cord injury associated with the immobilization of the 
patient leads to a decrease in bone mass. The bone loss greater is observed in the 6 months after the spinal cord injury, and stabilizing between 12-16 months after the same. The incidence of fractures oscillates between $1.5 \%$ and $6 \%$.

Objectives: To assess bone metabolism and bone fracture incidence in SPI patients.

Methods: Prospective study of SPI patients from the Spinal Cord Injury Unit of La Fe Hospital. In all cases densitometry, x-ray image, bone metabolism biomarkers and clinical evaluation have been performed according protocol. Statistical techniques was carried out using $R$ software 3.2.3, using mixed linear regression models.

Results: We studied 40 patients with SCI, $54 \%$ of them men and $46 \%$ women, with a mean age of 59.5 years (57.3- 63.5). The $58 \%$ of patients showed thoracic injuries, $48.6 \%$ paraparesis and $46.7 \%$ presented level C in Asia scale. The baseline study was performed in $100 \%$ of patients $(n=40), 65 \%$ in month $6(n=26)$, $30 \%$ in month $12(n=12)$, and in $22.5 \%$ in month $18(n=9)$. The $32.4 \%$ of patients received supplementation with calcium and vitamin $D$ at month $0,66.7 \%$ at month 6 and $100 \%$ at month 12 .

In month 6 , the $11.1 \%$ was treated with antiresorptive drugs.

An increase in vitamin $D$ values can be observed in the population with follow-up (values of 16.82 in month 0 to 39.33 in month 12), justified by the supplementation, and there is an increase in Calcium and a decrease in phosphorum values.

There was also a decrease in PTH levels in month 12 (32.3) compared to month 0 (34.08), as well as a decrease in bctx levels. Probably related to the increase of vitamin D.

Despite a decrease in the densitometric parameters at month 6, a slight recovery in bone mineral density at month 12 was observed. No bone fractures were seen during Follow-up in none of the patients.

Results from biochemical markers and densitometry are showed in the table

\begin{tabular}{lccc}
\hline Parameter & Month 0 & Month 6 & Month 12 \\
\hline & Mean (SD) & Mean (SD) & Mean (SD) \\
Calcium & $9.04(0.47)$ & $9.57(0.36)^{\star \star \star}$ & $9.39(0.34)^{\star \star \star}$ \\
Phosphorus & $3.83(0.62)$ & $3.42(0.45)^{\star \star \star}$ & $3.4(19.94)^{\star \star \star}$ \\
25OH Vitamin D & $16.82(10.4)$ & $37.27(15.52)^{\star \star \star}$ & $39.33(19.94)^{\star \star \star}$ \\
PTH & $34.08(21.74)$ & $36.16(19.46)$ & $32.3(12.6)$ \\
P1NP & $76.1(79.18)$ & $64.27(28.11)$ & $65.87(25.76)$ \\
BCTX & $0.94(0.46)$ & $0.62(0.87)^{\star \star}$ & $0.34(0.21)^{\star \star \star}$ \\
Lumbar spine tscore & $-0.24(1.58)$ & $-0.25(1.11)$ & $-0.3(1.27)$ \\
Femoral neck tscore & $-0.73(1.34)$ & $-1.35(1.03)^{\star \star}$ & $-1.26(1.25)^{\star \star}$ \\
Hip bone tscore & $-0.74(1.25)$ & $-1.56(1.29)^{\star \star \star}$ & $-1.52(1.4)^{\star \star \star}$ \\
\hline${ }^{*}$ ( & & &
\end{tabular}

${ }^{*} \mathrm{p}<0,05,{ }^{* *} \mathrm{p}<0,01,{ }^{* * *} \mathrm{p}<0,001$.

Conclusions: A high percentage of our patients with spinal cord injury has a vitamin $D$ deficiency. In addition, the lower levels are associated with cases where the mobility limitation is higher. As patients increase vitamin D values, a decrease in the bctx and PTH parameters is observed.

No fractures were detected during follow-up.

Disclosure of Interest: None declared

DOI: 10.1136/annrheumdis-2017-eular.5487

\section{AB0838 EPIDEMIOLOGY AND ANALYSIS OF FALLS IN PATIENTS DURING BALNEOTHERAPY IN DANUBIUS HEALTH SPA PIESTANY}

I. Rybar $^{1}$, V. Kolnikova ${ }^{2}$, T. Zimanova ${ }^{2}$, R. Gaspar ${ }^{2} .{ }^{1}$ Department of Rheumatology, National Institute of Rheumatic Diseases; ${ }^{2}$ Department of Physiotherapy, Danubius Health Spa, Piestany, Slovakia

Background: Patients falls in hospitals are frequent and undesirable complications that may lead to negative outcomes such as injuries, prolonged hospitalization and legal liability.

Objectives: To investigate the incidence and characteristics of patients hospitalized in the Health Spa Piestany.

Methods: Prospective analysis of falls in the group of in-patients of health spa during a year since 01.01 .2015 to 31.12 .2015 .

Results: Overall there were hospitalized in the health spa 25774 in-patients. The mean age of patients was $64,02 \pm 13,16$ years. There were women - 15301 , mean age $(63,61 \pm 11,57$ years) and men 10473 , with mean age $(64,43 \pm 9,22$ years). Falls were confirmed in 131 patients (women 94, mean age 68,55 $\pm 10,22$ years and men 37 males, mean age 70,39 $\pm 7,62$ years. Fractures have been confirmed in 19 patients, all of them non-vertebral. The ankle distortion were in 11 patients and lacerated wounds in 10 patients. There were collapse status in 8 and commotio cerebri in 1 patient. Spa therapy had to be discontinued only in 5 from 131 patiens due to falls. The annual incidence of falls reached 5,08 cases/1000 patients. In women there were - 6,14 cases/1000 women a year and in men 3,53 cases/1000 men a year. The annually incidence of fractures reached $0,69 / 1000$ pacients. The most common locations of falls were hotel rooms, especially bathtub in the bathroom (49\%), slightly less facilities for balneotherapy $(22 \%)$, the area outside the spa $(21 \%)$ and at least in the park area of health spa $8 \%$. Women and men who have suffered falls had significantly higher age and had also high rate of cardio-vascular morbidity (87\%).

Conclusions: The annual incidence of falls among patients in a balneotherapy reached to 5.08 cases per 1,000 patients per year. Incidence of falls in women was almost twice as high compared to men. The age of women and men with falls was significantly higher $(\mathrm{p}<0,001)$ compared to other patients and they also had higher rate of cardiovascular morbidity. The most common locations of falls were bathtubs in the bathroom.

References:

[1] Gazibara T. et al.: Psychogeriatrics. 2017 Jan 27. doi: 10.1111/psyg.12217.

[2] Cockayne S. et al.: PLoS One. 2017 Jan 20;12(1):e0168712.

[3] Tsonga T. et al.: Clin Orthop Surg. 2015 Dec;7(4):449-56.

Disclosure of Interest: None declared

DOI: 10.1136/annrheumdis-2017-eular.5278

\section{AB0839 THE EFFICACY AND SAFETY OF DENOSUMAB LOCAL EXPERIENCE)}

J. Al-Saleh, N. Salah, A.S. Hasan, S. Mohamad, F.A. ElBadawi, M. Khamashta. Rheumatology Department, Dubai Hospital, Dubai, United Arab Emirates

Background: Denosumab was introduce to the United Arab Emirates Market in 2013.Given the limited experience in using Denosumab in the region we have explored it efficacy and safety in daily practice.

Objectives: To assess the efficacy and safety of Denosumab in our practice.

Methods: Inclusion criteria: All patients received Denosumab in a dose of $60 \mathrm{mg}$ every six months. Underwent DEXA scan in Dubai Health Authority. Exclusion from analysis: Other Doses of Denosumab, who received less than 3 doses, and those with no follow up DEXA scan. Outcome measures: 1) Efficacy: Proposed Criteria for Assessing Clinical Response $[1,2]$. a) "inadequate": incident fracture and significant BMD decrease. b) "possible inadequate": incident fracture or significant BMD decrease. c) "appropriate": no fracture and stability or increase in BMD. 2) Safety: Reviewing the medical records and conduct patients interview for occurrence of the following adverse events. Statistical analysis: Descriptive statistical analysis, Graphpad Prism 6 was used.

Results: 143 patient identified. Out 139 patients 86 were eligible for analysis (See table 1). At baseline $39 \%$ had normal vitamin D level, $57 \%$ had insufficiency and $4 \%$ had deficiency. 20 of 86 did not undergo repeated DEXA scan. $9 \%$ had osteopenia and $91 \%$ had osteoporosis before initiating Denosumab in comparison $8 \%$ had normal bone mineral density, $45 \%$ had osteopenia and $47 \%$ post four injections of Denosumab. Table 2 summarize the comparison between the responders and non-responders. There was a significant positive correlation in the increase in bone mineral density among the responders at the femoral neck and the lumbar spine, $(r=0.56,95 \%$ confidence interval: $0.31-0.74, \mathrm{P}$-value $<0.0001$ No report of any of the adverse events 86 patients who completed 2 years or more on treatment.

Table 1. Patients characteristics at baseline

\begin{tabular}{lcc}
\hline Variables & Number & Percentage \\
\hline Age (yrs) median, (1st Quartile- 3rd Quartile) & $65,(58-67)$ & \\
$51-65$ & 48 & $35 \%$ \\
$>65$ & 76 & $55 \%$ \\
Female & 126 & $91 \%$ \\
UAE & 121 & $87 \%$ \\
Comorbidities & 72 & $52 \%$ \\
Received 4 Denosumab doses or more & 86 & $62 \%$ \\
DEXA scan in DHA at Baseline & 136 & $98 \%$ \\
Osteopenia & 12 & $9 \%$ \\
Osteoporosis & 124 & $91 \%$ \\
Fractures & 12 & $9 \%$ \\
\hline
\end{tabular}

Table 2. Comparison between the appropriate Response group "Responders" and inadequate response group "Non-responders"

\begin{tabular}{lccccc}
\hline Variables & Responders & Non-responders & Odds Ratio & P-value & $95 \% \mathrm{Cl}$ \\
\hline Number & 52 & 14 & & & \\
Rheumatic Diseases & 6 & 36 & 0.1 & 0.0001 & $0.05-0.2$ \\
Fracture & 0 & 3 & 0.2 & NS & \\
Pre-treatment fractures & 9 & 1 & 9.5 & 0.02 & $2.0-70$ \\
\hline
\end{tabular}

Conclusions: Denosumab was effective and safe in our patients. Long-term follow up is required to verify these findings in our population.

References:

[1] Diez-Perez and Gonzalez-Macias. Inadequate responders to osteoporosis treatment: proposal for an operational definition. Osteoporos Int. 2008 Nov;19(11):1511-6.

[2] E. M. Lewiecki \& N. B. Watts. Assessing response to osteoporosis therapy Osteoporos Int (2008) 19:1363-1368.

Disclosure of Interest: None declared

DOI: 10.1136/annrheumdis-2017-eular.6791 